\title{
Evaluation des déterminants de la valeur perçue d'un produit: Cas des faux cheveux en contexte africain
}

\author{
Donanfra Hubert TUO \\ Laboratoire des Sciences des Organisations (LSO) \\ Unité de Formation et de Recherche des Sciences Economiques et de Gestion \\ Université Félix Houphouët-Boigny, Abidjan (Côte d'Ivoire)
}

\begin{abstract}
Résumé : L'objectif de cet article est d'identifier les composantes de la valeur globale perçue dans l'utilisation des faux cheveux conformément aux typologies proposées par les spécialistes des études du comportement des consommateurs et d'en évaluer leurs poids. A cet effet, 25 entretiens individuels et 02 focus groups ont été menés auprès des femmes, auxquels s'est ajouté un mini questionnaire administré à 100 femmes. Les résultats montrent dans l'ordre d'importance que les bénéfices perçus composés des valeurs d'esthétique (beauté), d'expression de soi (estime de soi), d'hédonisme (plaisir) d'efficience, d'excellence (gain de temps) et d'utilité (protection) et les sacrifices monétaires perçus (prix) sont les antécédents de la valeur globale perçue de l'utilisation des faux cheveux.
\end{abstract}

Mots-clés : Valeur perçue, bénéfices perçus, sacrifices perçus, faux cheveux.

Digital Object Identifier (DOI): https://doi.org/10.52502/ijesm.v1i2.214 


\section{Introduction}

La notion de la valeur est « régulièrement mobilisée en sciences de gestion » et fait « l'objet d'une pluralité de regards disciplinaires sans réelle lecture fédératrice approfondie » (Brechet et Desreumaux, 1998). En effet, bien qu'elle soit employée en finance, comptabilité, gestion de production, gestion stratégique, gestion des ressources humaines et marketing, l'on constate l'absence de lecture transversale et fédératrice provoquée par l'absence d'articulation véritable des points de vue des différentes disciplines et d'un cadre théorique unifié. Le concept de valeur est polysémique aussi bien du fait de la diversité de ses sources théoriques (Rivière et Mencarelli, 2012), que de son usage au sien du marketing (Karababa et Kjeldgaard, 2014 ; Rivière et Mencarelli, 2012 ;).

$\mathrm{Au}$ cours de ces quarante dernières années, la valeur perçue a fait l'objet de nombreuses recherches (Mencarelli et Rivière, 2020) ; elle occupe une place significative dans les réflexions actuelles des praticiens et des chercheurs. Toutefois, le concept de valeur perçue reste problématique car il fait l'objet de diverses définitions dans la littérature en marketing (Sanchez-Fernandez et al, 2009) en fonction des auteurs et progressivement dans le temps. Plusieurs auteurs se sont attachés à mieux comprendre la valeur perçue des consommateurs sous deux principales approches, notamment du point de vue de l'entreprise et du point de vue du consommateur. Autrement dit les entreprises créent de la valeur pour les consommateurs et ceux-ci perçoivent la valeur parfois différente de la valeur réelle du bien ou service.

Ainsi « nous sommes passés d'un objectif de satisfaction des clients à celui de création de valeur » (Le Nagard et Michel, 2017). Ces derniers affirment que cette création de valeur devenue le maître mot dans les entreprises est sanctionnée par le niveau de prix acceptable pour une offre donnée. "Au-delà de la valeur fonctionnelle de l'offre du produit ou du service, le marketing met en avant de multiples autres sources de création de valeur ».

La compréhension du concept de valeur perçue ne réside pas seulement dans son aspect théorique. Son aspect managérial est récurrent, puisque les entreprises sont constamment confrontées au cas de positionnement perçu. Il convient donc de mettre en œuvre cette problématique au sein d'un terrain d'application permettant de répondre aux objectifs de mesure de la valeur. Les faux cheveux constituent un champ d'investigation intéressant pour étudier les apports d'une approche par la valeur. En effet, la chevelure est un ornement auquel les femmes accordent beaucoup d'importance et la symbolique qui y est attachée varie en fonction des époques et des lieux, car depuis son jeune âge, la femme y consacre assez de son temps pour son embellissement (Court, 2007). De plus, les ivoiriennes consacrent en moyenne $15 \%$ de leur revenu dans l'achat des faux cheveux (Emission « Made in Africa » de la RTI, diffusée le 20 avril 2018).

Compte tenu de l'intérêt de la prise de conscience d'une valeur expérientielle, l'objectif de cette recherche est d'identifier, à partir des définitions et des approches de classification, les composantes de la valeur globale perçue du point de vue du consommateur dans l'utilisation des faux cheveux et d'évaluer les préférences des consommateurs.

Ceci nous conduit à une réflexion chez les praticiens du marketing qui déploient leurs efforts afin de mieux appréhender les aspects et les spécificités du concept de la valeur perçue, dans un but de valorisation d'une part, et d'autre part de définir les motivations de création chez les consommateurs dans leur expérience de consommation en contexte ivoirien.

Dans le présent article, après avoir défini le cadre conceptuel de la recherche, nous exposerons ensuite notre démarche méthodologique. Et ensuite, nous présenterons les résultats et les commentaires. 


\section{Cadre conceptuel de la recherche}

\subsection{Concept de valeur perçus}

La notion de valeur est largement utilisée en sciences sociale. Plusieurs chercheurs en ont fait un centre d'intérêt dans leurs travaux. La valeur représente un paradigme important pour les études du comportement d'achat du consommateur (Day, 2002). Et la littérature en marketing a donné naissance à une diversité de conception de la valeur perçue (Aurier, Evrard et N'Goal, 2001). En économie, « La valeur est ce que les clients sont prêts à payer » (Porter, 1986). Mais en sociologie, la valeur est « une grandeur qui croit lorsque la satisfaction de l'utilisateur augmente ou que la dépense y afférant diminue » (Perrin, 2001, p 13), c'est un jugement porté par l'utilisateur sur la base de ses motivations et attentes.

Le produit peut être considéré comme un panier d'attributs procurant de l'utilité et de la satisfaction aux consommateurs (Lancaster, 1966), sources de création de valeur (Mencarelli et Rivière, 2012). En d'autres termes, la valeur est la représentation mentale du bénéfice que procurent les attributs; elle rend compte de la manière dont le consommateur perçoit le produit. Dans la même veine, Michel (2001) présente la valeur comme étant « la relation qui existe dans la tête d'un client ou usager entre le service rendu par un produit ou des fonctions sous-jacents ». C'est la raison pour laquelle Woodruff (1997) définit la valeur perçue comme " une préférence et une évaluation, faite par le client, des attributs du produit (ou de l'expérience), de ses performances et des conséquences de son utilisation (ou de son vécu) ».

\subsection{Formation de la valeur au sein du marketing}

La valeur est abordée en en marketing sous plusieurs angles et selon plusieurs approches : transactionnelle ou expérientielle (Parissier, 2007). Vue dans la perspective transactionnelle, la valeur perçue est appelée valeur d'achat et se forme avant l'acquisition du produit. Mais dans la perspective expérientielle, la formation de la valeur est faite à la suite d'une expérience de consommation ou de possession donnée (Bernoussi, N. et Sirieix, L., 2020) d'où l'appellation valeur de consommation. C'est l'expérience vécue par le consommateur ou l'acheteur qui produit et fait naître la valeur.

La chaîne moyens-fins peut être mobilisée pour expliquer la formation de la valeur en considérant que l'attribut produit apporte comme conséquence un bénéfice qui lui-même permet d'atteindre des valeurs instrumentales et terminale. En effet les attributs des produits sont vus comme des instruments pour atteindre diverses utilités fonctionnelles et psychosociales que le consommateur transforme pour satisfaire ses valeurs terminales (voir figure 1). Selon (Mencarelli et Rivière, 2012) un produit n'est pas acheté pour ses attributs, mais plutôt pour les bénéfices qu'il procure. Ces derniers constituent des sources de création et de formation de valeur au consommateur. En effet, la perception des bénéfices dépendants des attributs permet la perception de la valeur de l'offre (Valette-Florence et al, 2003) et favorise l'intention d'achat. Autrement dit le consommateur évalue les offres disponibles sur le marché, afin de faire des choix. Pour cela, il juge la qualité d'un produit se basant sur des attributs intrinsèques ou extrinsèques (Zeithaml, 1988) la valeur perçue est donc un jugement individuel et personnel. Ces attributs influencent sa perception et le rassurent dans ses choix.

Figure 1 : Processus de formation de la valeur

$\left.\left.\left.\left.\sum \begin{array}{c}\text { Attributs } \\ \text { Caractéristique objectives et } \\ \text { subiectives du produit }\end{array}\right\rangle \begin{array}{c}\text { Bénéfices } \\ \text { Conséquences fonctionnelles } \\ \text { et psycho-sociales }\end{array}\right\rangle\right\rangle \begin{array}{c}\text { Valeurs } \\ \text { Valeurs instrumentales } \\ \text { et finales }\end{array}\right\rangle$

Source : Adapté de la littérature 
Pour appréhender la valeur perçue d'un produit du point de vue du consommateur, deux courants de pensée se distinguent : les approches traditionnelles de la valeur et les approches expérientielles et interactionnistes.

\subsection{Approche traditionnelle cognitiviste de la valeur perçue}

Cette approche traditionnelle inspirée de la théorie économique, considère le consommateur comme un individu rationnel à la recherche de la maximisation de l'utilité de sa consommation et donc de sa satisfaction. Cette maximisation obéit à une logique calculatoire formulée en termes de ratio coûts/bénéfices (Zeithaml, 1988), autrement dit ratio achat/usage. Ainsi donc ce courant de pensée « classique » oppose deux conceptions de la valeur perçue, à savoir la valeur d'achat et la valeur d'usage.

\subsubsection{La valeur d'achat}

Toute marchandise possède une valeur d'échange. Dans cette acception, la valeur d'achat ou valeur d'échange, est le bénéfice ou avantage que le client tire de la consommation d'un produit. La valeur d'achat est centrée sur le moment de l'acte d'achat et l'évaluation des attributs du produit; elle résulterait ainsi d'un l'arbitrage entre les bénéfices ou avantages attendus de la consommation et les coûts ou les sacrifices consentis pour l'acquisition du bien.

\subsubsection{La valeur d'usage}

Woodruff (1997) définit la valeur d'usage comme étant «la préférence et l'évaluation du consommateur des attributs du produit (ou de l'expérience), de ses performances et des conséquences de son utilisation (ou de son vécu) facilitant ou bloquant l'atteinte des objectifs et des finalités que le client désire atteindre dans les situations d'usage ou de consommation ». Autrement dit, la valeur d'usage ou valeur de consommation, est la valeur que le consommateur tire d'une expérience de consommation c'est-à-dire les avantages attendus, l'utilité et les bénéfices. Celle-ci suggère la valorisation subjective du produit ou du service en fonction de l'utilité ou la satisfaction que le consommateur retirerait ou espèrerait en retirer. A partir de la notion de valeur d'usage, les consommateurs seront en mesure d'identifier l'usage des produits et des services afin d'en évaluer leur pertinence et de les valoriser. Cette conception correspond à une approche pluridimensionnelle, qui intègre les dimensions affective, expérientielle et situationnelle de la valeur (Aurier et al., 1998).

Dans l'approche traditionnelle, les avantages attendus s'expriment en termes de facilité d'utilisation $\mathrm{du}$ produit, utilisation sans risque, appartenance à un groupe, tandis que les sacrifices font allusion au prix à payer, à l'effort consenti, au temps employé pour acquérir le bien. Zeithaml (1988) définit alors cette conception de la valeur comme "l'évaluation globale par le consommateur de l'utilité d'un produit, sur la base de ses perceptions de ce qui est reçu et de ce qui est donné ». Quant à Aurier, Evrard et N'Goala, (2004), la valeur perçue est définie comme étant le résultat de la confrontation entre les bénéfices perçus et les coûts perçus ou sacrifices.

\subsection{Approches expérientielles et interactionnistes de la valeur perçue}

\subsubsection{Approche selon Holbrook (1994)}

Holbrook (1994) propose une classification des valeurs de consommation à partir de trois critères :

- le premier critère est l'orientation. Elle oppose l'orientation vers soi (fonction de l'intérêt personnel) à l'orientation vers les autres (famille, amis, collègues, société, divinité...),

- le deuxième critère est lié au caractère intrinsèque ou extrinsèque de la valeur de consommation. La valeur est intrinsèque lorsque l'expérience de consommation associée au 
produit est appréciée en tant que telle. A l'inverse, la valeur est extrinsèque lorsque l'expérience de consommation est un moyen pour atteindre des fins qui lui sont extérieures,

- enfin le troisième critère oppose le caractère actif (selon que le consommateur manipule physiquement ou mentalement l'objet) au caractère réactif de l'expérience de consommation (selon qu'il répond positivement).

Holbrook en combinant ses trois critères délimite huit domaines de compréhension de la valeur (voir tableau 1).

Tableau 1 : Typologie de la valeur de Holbrook

\begin{tabular}{|c|c|c|c|}
\hline \multicolumn{2}{|l|}{ Valeur } & Extrinsèque & Intrinsèque \\
\hline \multirow[t]{2}{*}{$\begin{array}{l}\text { Orientation } \\
\text { vers soi }\end{array}$} & Active & $\begin{array}{c}\text { Efficience } \\
\text { praticité, justesse, } \\
\text { convivialité }\end{array}$ & $\begin{array}{c}\text { Jeu } \\
\text { divertissement, } \\
\text { fantaisie, hasard, } \\
\text { transgression }\end{array}$ \\
\hline & Réactive & $\begin{array}{c}\text { Excellence } \\
\text { qualité, efficacité, } \\
\text { potentialité }\end{array}$ & $\begin{array}{c}\text { Esthétique } \\
\text { beauté, sensualité }\end{array}$ \\
\hline \multirow{2}{*}{$\begin{array}{l}\text { Orientation } \\
\text { vers les autres }\end{array}$} & Active & $\begin{array}{c}\text { Statut } \\
\text { succès, gestion de } \\
\text { soi, image }\end{array}$ & $\begin{array}{c}\text { Ethique } \\
\text { vertu, justice, } \\
\text { morale }\end{array}$ \\
\hline & Réactive & $\begin{array}{c}\text { Estime } \\
\text { réputation, } \\
\text { matérialisme, } \\
\text { possession }\end{array}$ & $\begin{array}{c}\text { Spiritualité } \\
\text { foi, extase, sacré, } \\
\text { magie }\end{array}$ \\
\hline
\end{tabular}

Source : Holbrook M. (994)

\subsubsection{Approche selon Holt (1995)}

L'approche de Holt (1995) conçoit que pour le consommateur, l'expérience est source de valeur, parce qu'elle s'inscrit dans l'action. Holt propose une classification des pratiques de consommation sur la base de deux critères dichotomiques. Le premier est basé sur l'objectif de la pratique qui peut être instrumental ou autotélique. Le second critère, «structure de la pratique », concerne la nature sociale ou individuelle de l'objet de consommation; il oppose les pratiques orientées vers une interaction avec l'objet aux pratiques orientées vers une interaction avec d'autres personnes.

Le croisement de ces deux critères donne naissance à quatre familles de significations (Confère tableau 2):

- l'« expérience» (autotélique/orientée objet), qui correspond à la construction d'un cadre interprétatif permettant de donner du sens aux objets de la consommation et de les apprécier,

- le «jeu» (autotélique/orientée interpersonnel), qui est le résultat de l'utilisation des objets pour partager l'expérience de consommation avec les autres ou enrichir son caractère divertissant,

- 1' «intégration » (instrumentale/orientée objet), qui correspond aux processus d'utilisation des significations symboliques de l'objet pour l'intégrer dans le soi étendu (Belk, 1988 cité par Aurier et al. 2004) ou à l'inverse réorienter le soi en fonction de l'objet (Solomon, 1983 cité par Aurier et al. 2004), 
- la «classification» (instrumentale/orientée interpersonnel), qui est le résultat de l'utilisation des objets dans le positionnement par rapport aux autres, en particulier en termes de communication, affiliation et distinction.

Tableau 2 : Les métaphores des pratiques de consommation de Holt

\begin{tabular}{|l|l|l|l|}
\hline \multicolumn{2}{|c|}{ Valeur } & Objectif de l'action \\
\cline { 3 - 4 } & Autotélique & Instrumentale \\
\hline \multirow{2}{*}{$\begin{array}{l}\text { Structure de } \\
\text { l'action }\end{array}$} & Objet & Expérience & Intégration \\
\cline { 2 - 4 } & Interpersonnelle & Jeu & Classification \\
\hline
\end{tabular}

Source : Holt D. B. (1995)

\subsubsection{Approche selon Lai (1995)}

L'approche de Lai (1995) est complémentaire des travaux antérieurs axés sur les bénéfices perçus lors de la consommation. Il s'agit des bénéfices :

- fonctionnels, correspondant à la performance utilitaire, physique et pratique du produit et dérivant de ses attributs tangibles et concrets,

- sociaux, relatifs aux associations du produit avec un statut et une classe sociale,

- émotionnels ou affectifs, résultant de la capacité du produit à susciter des sentiments,

- épistémiques, faisant référence à la capacité du produit à satisfaire la curiosité, le désir de connaissance ou de nouveauté,

- conditionnels ou situationnels, correspondant à la capacité du produit à satisfaire les contraintes de la situation et augmenter d'autres types de bénéfices,

- hédoniques, liés à la capacité du produit à produire du plaisir, de l'amusement,

- esthétiques, qui associent la consommation du produit à la beauté et l'expression personnelle,

- holistiques, issus de la complémentarité et la cohérence du produit avec la constellation des produits déjà consommés ou possédés,

En intégrant cette approche aux deux dichotomies présentées précédemment (« orientation vers soi ou vers les autres, « intrinsèque/extrinsèque ») on aboutit à la catégorisation où il est impossible de classer deux bénéfices : holistique et situationnel (Confère tableau 3).

Tableau 3 : Essai d'intégration des approches de la valeur de Lai

\begin{tabular}{|l|c|c|}
\hline \multicolumn{1}{|c|}{ Valeur } & Extrinsèque & Intrinsèque \\
\hline Orienté vers soi & Epistémique & $\begin{array}{c}\text { Hédonique } \\
\text { Affectif } \\
\text { Esthétique }\end{array}$ \\
\hline $\begin{array}{l}\text { Orienté vers les } \\
\text { autres }\end{array}$ & Social \\
\hline \multicolumn{2}{|l|}{ Non classé : Holistique et Situationnel } \\
Source : Lai A. W. (1995)
\end{tabular}

\subsubsection{Approche selon Evrard et Aurier (1996)}

Evrard et Aurier (1996) ont auparavant proposé une typologie de sept composantes de la relation personne-objet. En effet, ces auteurs se sont basés sur la théorie fonctionnelle des attitudes pour étudier les dimensions de la relation entre une personne et un objet. La théorie stipule que l'individu développe des attitudes pour atteindre des buts, autrement dit, il s'adapte à son environnement tout en protégeant la représentation qu'il a de lui-même. 
Sur la base de cette théorie, la classification d'Evrard et Aurier (1996) est faite à la fois sur une dimension expérientielle identifiant les valeurs hédonique, stimulation expérientielle, et pratique sociale et une autre dimension non expérientielle avec les valeurs connaissance, expression de soi, lien social et recherche d'information. En intégrant leur approche aux deux dichotomies présentées précédemment : «orientation vers soi ou vers les autres, «intrinsèque/extrinsèque » ils aboutissent à l'organisation du tableau 4.

Tableau 4 : Typologie de la valeur d'Evrard et Aurier

\begin{tabular}{|l|c|c|}
\hline Valeur & Extrinsèque & Intrinsèque \\
\hline Orienté vers soi & $\begin{array}{c}\text { Connaissance } \\
\text { Recherche d'information }\end{array}$ & $\begin{array}{c}\text { Hédonique } \\
\text { Stimulation expérientielle }\end{array}$ \\
\hline $\begin{array}{l}\text { Orienté vers les } \\
\text { autres }\end{array}$ & $\begin{array}{c}\text { Expression de soi } \\
\text { Lien social }\end{array}$ & Pratique sociale \\
\hline
\end{tabular}

Source : Evrard Y. et Aurier P. (1996)

\subsubsection{Approche selon Aurier, Evrard et N'Goala (2004),}

Dans leur recherche, Aurier, Evrard et N'Goala (2004) se sont engagés à comparer les approches de Holbrook (1994), Holt (1995), Lai (1995), et Evrard et Aurier (1996) dans le but de permettre leur intégration. Pour cela, les auteurs ont été amenés à retenir deux critère qui leur "semblent centraux dans les classifications proposées : d'une par le caractère extrinsèque/intrinsèque de la consommation et d'autre part son orientation vers soi/vers les autres » tout en écartant le critère actif/réactif de la typologie proposée par Holbrook (1994).

Leur analyse de la littérature et son intégration selon les deux critères choisis (« extrinsèque/intrinsèque » et « orientation vers soi/vers les autres »), ainsi que la mise en évidence de la théorie fonctionnelle des attitudes a permis de caractériser quatre familles de composantes de la valeur (voir tableau 5) : instrumentale, hédonique, communication et spirituelle.

La valeur instrumentale, correspond à la famille où la valeur utilitaire (déjà développée) et la valeur connaissance sont distinguées. La valeur connaissance est assimilable à la motivation des pratiques culturelle (Aurier et Passebois, 2002) ; elle correspond au désir de connaissance, d'enrichissement et de structuration de l'environnement afin d'en tirer le meilleur parti (Katz, 1960) et à la recherche continue d'information, porteuse de stimulation pour l'individu (Steenkamp et Baumgartner, 1992). Cette famille est orientée vers soi et extrinsèque.

La valeur hédonique correspond à la capacité de l'expérience à stimuler les sens de l'individu (Evrard et Aurier, 1996). Hirschman et Holbrook (1982) ont fait correspondre la dimension plaisir-amusement. Cette famille de valeur est orientée vers soi et intrinsèque.

La valeur de communication est la famille dans laquelle les auteurs se sont proposé « de distinguer l'expression des valeurs et le lien social, qui correspond à la fonction d'ajustement social ». Elle «appréhende la capacité du produit ou service à projeter l'expression de soi et à jouer un rôle dans la communication sociale en tant que reflet de la personnalité ». La valeur communication correspond à une famille de valeur orientée vers les autres et extrinsèque.

La spiritualité participe à la défense de l'ego en vue de maintenir l'estime de soi en fonction du regard des autres. Selon Evrard et Aurier (1996), on peut trouver dans la valeur spirituelle la pratique sociale. La valeur spirituelle est orientée vers les autres et intrinsèque. 
Tableau 5 : Typologie synthétique des composantes de la valeur d'Aurier, Evrard et N'Goala

\begin{tabular}{|l|c|c|}
\hline Valeur & Extrinsèque & Intrinsèque \\
\hline Orienté vers soi & $\begin{array}{c}\text { Valeur instrumentale } \\
\text { (utilitaire, connaissance) }\end{array}$ & $\begin{array}{c}\text { Valeur hédonique } \\
\text { (plaisir-amusement, stimulation } \\
\text { expérientielle) }\end{array}$ \\
\hline $\begin{array}{l}\text { Orienté vers les } \\
\text { autres }\end{array}$ & $\begin{array}{c}\text { Communication (expression de } \\
\text { soi, lien social) }\end{array}$ & $\begin{array}{c}\text { Valeur spirituelle, pratique sociale } \\
\text { Source : Aurier P., Evrard Y. et N'Goala G. (2004) }\end{array}$ \\
\hline
\end{tabular}

\subsection{Modèle intégrateur de la valeur perçue}

Aurier et al (2004) proposent un modèle intégrateur expliquant la valeur globale d'un produit comme résultant de la «confrontation entre les bénéfices et les sacrifices associés à la consommation ». Zeithaml (1988) la définit comme "l'évaluation globale de l'utilité d'un produit fondé sur les perceptions de ce qui est reçu et donné » ou encore comme «le rapport entre les bénéfices et les sacrifices perçus ».

Figure 2 : Modèle intégrateur de la valeur perçue

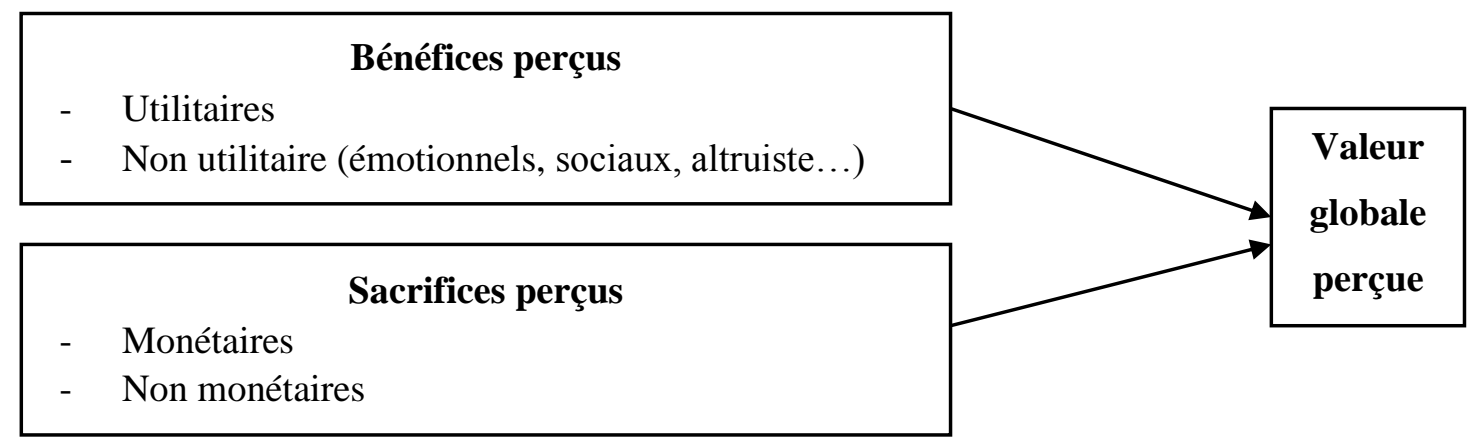

Source : Aurier P., Evrard Y. et N'Goala G. (2004)

\section{Approche méthodologique}

\subsection{Choix de l'échantillon}

Le choix de la femme en général comme cible de notre enquête est dû au fait que d'une part, le cheveu est pour elle un puissant symbole de féminité et de séduction (Rondou, 2014); et d'autre part, le rapport d'intimité qu'elle a avec les cheveux (Dibie, 2013). La particularité des africaines porte sur le fait qu'elle « utiliseraient neuf fois plus de produits capillaires que leurs homologues caucasiennes » (Peretti et Delannoy, 2010, p. 289). Autant dire que les femmes aux cheveux crépus sont des consommatrices idéales de faux cheveux. Le faux cheveu est en lui-même un substitut ou complément de cheveu naturel, communément appelé « mèche » par nos répondants.

\subsection{Choix méthodologique}

Pour d'atteindre l'objectif de notre étude qui est d'explorer les perceptions de la valeur des utilisatrices de faux cheveux, nous avons mené successivement une étude documentaire, des observations, des entretiens et une étude quantitative non approfondie. 


\subsubsection{Etude documentaire}

La recherche d'informations commence en général par une étude de la documentation existante. Cette étude documentaire, nous a conduit à parcourir les magazines féminins sur la coiffure, les archives sur «Youtube» (sur le Business des mèches datant du 26 décembre 2017 et le 20 avril 2018) de l'émission " Made in Africa » de la chaîne nationale ivoirienne (RTI) ; son objectif est de nous familiariser au phénomène des faux cheveux. Les résultats de notre recherche documentaire nous informent que les africaines dépensent environ 7 milliards de dollars par an dans l'achat des faux cheveux dont 500000000 F CFA par an pour les ivoiriennes.

\subsubsection{Observation}

Selon Gavard-Perret et al. (2012, p. 166), « l'observation peut être définie, comme une technique de collecte de données primaires visibles et audibles ». Conformément à Jones (2000), notre action a été de mener une observation passive en observant les « comportements non verbaux » des femmes dans les rues, dans les points de vente des faux et les salons de coiffure pour femmes.

Nos observations nous montrent que le type de coiffure portée par une femme est expressif, elle nous renseigne sur le pouvoir d'achat, l'occupation, l'âge, le caractère introverti ou extraverti de l'individu, la durée de la coiffure, etc. En moyenne quatre femmes africaines sur cinq portent des faux cheveux (à des prix et qualités différents, des couleurs variées, des coiffures diversifiées...) pour aller au travail, à des baptêmes, à des anniversaires, à des mariages, à des fêtes et d'autres événements joyeux de tout genre. De plus deux tendances apparaissent : une catégorie de jeunes filles (moins de 30 ans) fascinées des coiffures volumineuses et longues qui tombent dans le dos et les autres plus âgées optent pour des coiffures courtes et peu importantes, qui tombent à la limite sur les épaules.

\subsubsection{Entretien de groupe}

Conformément aux deux tendances révélées par nos observations, nous avons procédé à la formation de deux focus groups de deux heures environ: un premier groupe composé de huit jeunes lycéennes et étudiantes de moins de 30 ans et le second groupe de dames de 30 ans et plus, toutes tendances confondues. Ces entretiens nous a permis d'obtenir des informations sur l'usage des faux cheveux, l'attitude et la perception des femmes.

\subsubsection{Entretiens individuels}

Ouattara (2003) précise que les entretiens individuels sont ceux qui sont les plus utilisés en Afrique. Elles ont pour objectif de relever les principales composantes de la valeur perçues à l'issue d'une expérience d'utilisation de faux cheveux en nous appuyant sur les typologies de la valeur perçue parcourues dans notre revue de littérature. Le but est de comprendre dans quelle mesure ces différentes typologies pourraient-elles s'appliquer au domaine de l'utilisation de faux cheveux, et donc d'identifier les sources et les principaux fondements des différents types de valeur à travers les discours des interviewés. À cet effet, nous avons réalisé des entretiens semi-directifs auprès de vingt (20) femmes. Ces entretiens d'une durée moyenne d'une demi-heure par enquête ont été menés à l'aide d'un guide d'entretien sur les motifs d'achat et de type de valeurs attendues. 


\subsubsection{Entretien d'experts}

Les entretiens d'experts ${ }^{1}$ viennent compléter les informations des entretiens individuels semi-directifs et les entretiens de groupe afin d'approfondir d'autres réalités de notre domaine de recherche. Nos experts au nombre de cinq (05) individus sont des personnes ressources qui occupent des fonctions saillantes dans la chaîne de distribution des faux cheveux, il s'agit de deux (2) commerçants et de (03) coiffeuses. Nos entretiens ont consisté en des discussions libres d'une durée moyenne de 40 min qui ont essentiellement porté sur deux thèmes : la connaissance de l'environnement et le phénomène des faux cheveux d'une part et d'autre part sur les motivations et les attentes des femmes.

\section{Résultats et discussion}

\subsection{Analyse préliminaire des entretiens}

L'analyse des discours fait ressortir quatorze (14) lots de signification à savoir " se faire voir », " affirmer sa personnalité », « se faire belle pour soi-même », « se faire belle pour autrui », « tendance trop occupée », «tendance à faire des économies de temps », « protection des cheveux », «faire pousser les cheveux », «tendance à aimer et porter », «tendance à apprécier sur autrui », « contraint à utiliser », « tendance à détester les faux cheveux », « prix élevé » et «se faire plaisir ». Sur la base des propositions d'Aurier, Evrard et N'Goala (2004) et de Zeithaml (1988) expliquant la valeur globale comme la confrontation entre les bénéfices perçus et les sacrifices perçus, nous avons partagé les thématiques en deux groupes.

\subsection{Bénéfices perçus}

Conformément à la typologie des bénéfices perçus à la consommation d'Aurier, Evrard et N'Goala (2004) et de Holbrook (1994) et l'analyse des verbatim des experts et des consommatrices de faux cheveux révèle des bénéfices d'esthétique (beauté), bénéfices d'estime (estime de soi), bénéfices utilitaires (protection des cheveux), bénéfices d'efficience et d'excellence (gain de temps) et des bénéfices hédoniques (plaisir).

\subsubsection{La beauté}

La rechercher permanente de la beauté semble être le quotidien de toutes les femmes. Le cheveu possède une valeur esthétique (Rondou 2014), c'est pourquoi elles sont enquête de tout produit susceptible d'atteindre leur objectif. Les normes de beauté actuelles poussent les femmes à consommer toujours davantage de produits et techniques esthétiques mis sur le marché (Djoma et Tsapi, 2012) comme les faux cheveux. A la question pour quelle principale raison les femmes portent elles les faux cheveux, les personnes interrogées avouent que «la plupart des femmes qui achètent les mèches disent qu'elles veulent se rendent belle (commerçante 2) ». A travers les techniques de manipulation des mèches de cheveux, les coiffeurs sont mis à l'épreuve car aujourd'hui, beauté et coiffure sont indissociables car la coiffure accompagne les critères de choix des reines de beauté (Monjaret et Tamarozzi, 2005). L'objectif principale des coiffeuses c'est de rendre les femmes belle «il n'y a pas de commentaire une belle coiffure avec les mèches ne peut que rende la femme encore plus belle (coiffeuse 1) ». Les femmes dans leur majorité voient les faux cheveux comme des accessoires de beautés, "les mèches c'est comme les bijoux, il y en a tout une variété pour affirmer votre beauté; vous avez les tresses, les nattes et les perruques que j'aime bien (consommatrice 14)", "j'aime

\footnotetext{
${ }^{1}$ Lambin (1990) définit l'expert comme étant toute personne qui, en raison de sa fonction, détient une information privilégiée ou particulièrement fiable. Selon Evrard et al. (1997), les experts sont des personnes qui, par leurs fonctions, leurs spécialités ou leurs expériences détiennent une expertise qui permettra de mieux comprendre le problème posé, ses différents aspects et les points critiques à prendre en compte.
} 
toujours me faire belle et je veux toujours être belle (...) avec les mèches c'est la solution rapide (consommatrice 6) », "Or aujourd'hui, pour se faire belle et "être à la page" il faut forcement les mèches (consommatrice 3) ». Le jugement de d'autres femmes porte sur l'œil extérieur, exemple: «mon marié trouve que je suis plus belle quand je porte les mèches (consommatrice 10) », « on me dit qu'avec les mèches on ne voit pas la grosse cicatrice qui est située sur ma tête (consommatrice 4) », « Je me fais belle pour mon mari, car il est toujours heureux quand je reviens du salon méchée (consommatrice 10)».

\subsubsection{L'estime de soi}

Selon Banister et Hogg (2004) l'estime de soi en comportement du consommateur influence la motivation et la décision d'achat. Effectivement l'achat des faux cheveux est motivé chez certaines femmes par le désir de changement de personnalité ; elles avancent : "pour être mannequines ou topmodèles pour une africaine, les cheveux crépus courts lissés doivent être allongés par des mèches (consommatrice 18) », "Je veux avoir une chevelure de star et avec les mèches ça marche » et "les mèches changent mon "look" et me rapprochent de mon idéal (consommatrice 8)».

Les soins que les femmes apportent à leurs cheveux sont influencés par leur estime de soi. Cela se vérifie par l'importance des budgets que d'autres femmes allouent au soin des cheveux; pour elles le coût n'a pas d'importance, "Je ne regrette pas de payer cher pour avoir des mèches humains, et payer encore cher pour les entretenir, parce que c'est la qualité, c'est la classe et ça te distingue quoi ! (consommatrice 7) ». Pour cette catégorie le prix impose le respect, ainsi «quand tu te coiffes avec les mèches humains, tu es respecté parce que tu n'es pas n'importe qui! (consommatrice 12)», «Ce n'est pas n'importe qui, nous qui portons les mèches Humains (consommatrice 7)».

Une autre catégorie de femmes encore plus jeunes et extraverties jouent sur les atouts qu'elles ont tel que le teint clair en Afrique noir, pour elles «quand vous êtes clairs, vous portez des mèches de couleur brun ou blonde et vous apparaissez inaperçus comme une blonde (consommatrice 5) ».

Dans cette quête d'une estime de soi, les jeunes africaines s'appuient sur un référentiel qu'elles sont loin d'avoir naturellement, celui des cheveux caucasiens «lisses et longs». Elles avouent que «les tissages et les tresses (leur) tombent sur le dos à la manière des stars d'Hollywood (coiffeuse 2)». Il n'en demeure pas moins pour les artistes et chanteuses qui «préfèrent les mèches de couleur blanche, verte, rouge, bleu, violet, (tout ce qui est loin de la couleur naturelle) pour affirmer leur présence partout où elles passent (commerçante 1)». À contrario, des femmes responsables plus réservées avouent aimer les couleurs simples comme le «noir, roux ou noir avec les extrémités roux ou marron couleur chocolat (consommatrice 2)».

\subsubsection{La protection des cheveux}

Pour les femmes en général la chevelure est une partie sacrée de leur corps, elles ont une relation d'intimité avec leurs cheveux; les entretenir et y prendre grand soin fait partie de leur quotidien. Comparativement au cheveu asiatique et caucasien, la texture du cheveu crépu présent une certaine fragilité quant aux cassures. Les utilisatrices de faux cheveux estiment qu'ils «empêchent les cheveux de se casser (consommatrice 15) » et pour donner une jeunesse aux cheveux naturels elles affirment que «des tresses ou des nattes avec les mèches font pousser très vite les cheveux (consommatrice 9) ».

Pour aller à l'encontre des détracteurs du port des faux cheveux qui présente les comme origine des alopécies certaines femmes affirment que «porter les perruques est un moyen pour laisser le cuir 
chevelu se reposer (consommatrice 3)", " les mèches jouent le même rôle qu'un chapeau (consommatrice 1)».

\subsubsection{Le gain de temps}

La pression temporelle, le manque de temps, sa mauvaise gestion ou l'activité quotidienne des individus sont autant de facteurs qui poussent les femmes à opter pour les faux cheveux. En effet une coiffure avec les faux cheveux peut durer deux semaines à plus de trois mois permettant ainsi à la femme de mettre à profit le temps énorme qu'elles consacrent à la coiffure. A cet effet on peut entendre les propos suivants : "quand tu te tresses bien avec les mèches, ta coiffure peut durer quatre mois voire même six mois (coiffeuse 3) », "avec les mèches, tu n'es plus obligé de faire les vas et viens chez les coiffeuses (consommatrice 1)», "Je descends tard du travail et je remonte tôt, je n'ai pratiquement pas le temps de passer à la coiffure c'est pourquoi j'achète tranquillement mes perruques (consommatrice 3)», " peigner mes cheveux naturels, les entretenir ou aller à la coiffure me prend assez de mon temps, c'est pourquoi j'enfile tout simplement mes perruques (consommatrice 13) », "avec les mèches, surtout les perruques, je ne perds pas assez de temps les matins pour faire ma coiffure (consommatrice 11) ", "les coiffures avec les mèches me font gagner plus de temps car elles durent plus sur ma tête que les coiffures avec mes cheveux naturels (consommatrice 17) ».

Des propos tirés de nos entretiens du genre, "Mon travail ne me laisse pas assez de temps, avec les perruques je ne perds plus de temps pour aller au salon (consommatrice 3) », "Une fois que je fais des tissages ou des tresses avec les mèches, la prochaine coiffure sera après trois mois et je gagne du temps (consommatrice 9) ", "les femmes, comme beaucoup le pensent déjà, utilisent les faux cheveux pour gagner du temps et vaquer à leurs activités, et dans certains cas pour éviter la chute des cheveux (commerçante 2) », présentent ainsi les faux cheveux avec la double fonction de protection des cheveux naturels et instrument de gestion du temps.

\subsubsection{Le plaisir}

L'utilisation des faux cheveux est pour certaines femmes une source de plaisir; d'autres y retire du sensationnel. Cela peut s'apercevoir à travers les propos suivants: «les différentes coiffures et couleurs de mèche accompagne le décor des évènements festifs; comme c'est beau et ça fait plaisir (coiffeuse 3) », « tu peux faire la remarque, les jours de fêtes, les anniversaires, les mariages, le 24 décembre ou le 31 décembre, toutes les femmes sont en mèche y comprise la mariée et ça fait beau (consommatrice 12) », «moi particulièrement je n'aime pas trop les mèches, mais ça me fait plaisir de les porter à mes petites filles (consommatrice 20)».

\subsection{Sacrifices perçus}

Les sacrifices perçus relevés par l'analyse des discours des interviewés sont de nature monétaire car le temps perdu au cours du choix de la marchandise et celui perdu chez la coiffeuse relève du plaisir ; les femmes prennent plaisir à passer du temps au shopping et à la coiffure. Un extrait de l'émission « Made in Africa » diffusée le 05 avril 2018 sur les ondes de la télévision nationale ivoirienne, mentionne que les femmes dépensent pour ces faux cheveux en moyenne $25 \%$ de leur revenu, occupant ainsi le troisième $\left(3^{\mathrm{e}}\right)$ post de leur budget après l'alimentaire et le vêtement. En effet, les femmes ne lésinent pas sur les moyens pour se rendre belle afin d'affirmer leur statut social (« Je ne regrette pas payer cher pour avoir des mèches humains, et payer encore cher pour les entretenir... (Consommatrice 7)»). 


\subsection{Correspondance et évaluation des valeurs perçues}

Les résultats dégagés de l'analyse thématique opérée sur le discours des experts et des consommateurs ont été comparés aux types valeurs inventoriés dans la littérature en vue de faire des correspondances. Les valeurs perçues identifiées correspondent à ceux attendus, à quelques exceptions près comme on peut le constater au tableau 6.

Tableau 6 : Correspondance des valeurs identifiées

\begin{tabular}{|l|l|l|}
\hline \multicolumn{2}{|l|}{ Types de valeur } & Valeurs identifiées \\
\hline Bénéfice & Esthétique & Beauté \\
\cline { 2 - 3 } & $\begin{array}{l}\text { Communication } \\
\text { (expression de soi) }\end{array}$ & Estime de soi \\
\cline { 2 - 3 } & Hédonique & Plaisir \\
\cline { 2 - 3 } & Efficience, excellence & Gain de temps \\
\cline { 2 - 3 } & Utilitaire & Protection \\
\hline Sacrifice & Monétaire & prix \\
\hline
\end{tabular}

Source : Auteur

Notre second objectif est de d'évaluer le poids des valeurs perçus identifiées dans l'utilisation des faux cheveux. Les résultats de notre sondage nous a permis de calculer les moyennes.

Tableau 7 : Moyenne des valeurs perçues identifiées

\begin{tabular}{|l|l|l|l|l|}
\hline & $\mathrm{N}$ & Minimum & Maximum & Moyenne \\
\hline Beauté & 100 & 2 & 5 & 4,68 \\
Estime de soi & 100 & 2 & 5 & 4,57 \\
Plaisir & 100 & 1 & 5 & 4,54 \\
Gain de temps & 100 & 2 & 4 & 3,28 \\
Protection & 100 & 1 & 4 & 2,35 \\
Prix & 100 & 1 & 3 & 1,89 \\
\hline
\end{tabular}

Source : Auteur à partir des résultantes de l'enquête

Les valeurs «beauté », «estime de soi » et «plaisir» ont dans l'ordre de présentation un positionnement largement supérieur à la moyenne. Ces différents résultats signifient que les femmes trouvent dans les faux cheveux une expression forte d'affirmation de leur beauté et de l'estime qu'elles ont d'elle mêmes, auxquelles s'ajoute le plaisir qu'elles ressentent dans l'utilisation des faux cheveux.

On constate cependant un positionnement moyen pour la valeur « Gain de temps » qui tourne autour de la moyenne 3. Quant aux valeurs «protection» et «Prix » elles ont un très faible positionnement eu égard leurs moyennent qui tournent autour de 2.

\section{Conclusion}

Nous attendions de cette étude exploratoire des résultats en termes de bénéfices perçus et de sacrifices perçus qui forment la valeur globale perçue des faux cheveux d'abord. Ensuite, la classification de ces valeurs perçus conforment aux typologies relevées par les spécialistes de études du comportement des consommateurs. Et enfin, des résultats en terme évaluation du poids des valeurs perçus identifiées. 
Finalement, nos résultats de notre rechercher montrent que la décision d'utilisation des faux cheveux des femmes est motivée par une perception globale qu'elles ont du produit en question. Cette perception globale est déterminée certaines valeurs qu'elles perçoivent. En effet, à travers la notion de la valeur perçue, nos résultats nous ont permis d'identifier la beauté, l'estime de soi, le plaisir, le gain de temps et la protection comme bénéfice d'une part et le prix comme sacrifice d'autre part.

Conformément à la typologie de la valeur perçue selon nos spécialistes à la matière, nos résultats mettent également en relief le niveau de motivation dans l'utilisation des faux cheveux. En effet, le niveau d'utilisation des faux cheveux est très peu influencé par le sacrifice monétaire (prix élevé) que par les bénéfices. Ceux sont respectivement les bénéfices esthétiques (beauté), les bénéfices communication ou expression de soi (estime de soi) et les bénéfices hédoniques (plaisir) qui ont un fort pouvoir d'influence décisionnelle. Les bénéfices efficience et excellence (gain de temps) ainsi que Les bénéfices utilitaires (protection) sont à un moindre niveau dans le poids de la décision.

Les apports managériaux de cette recherche sont à deux niveaux. Pour bénéficier d'une part d'une évaluation globale positive d'un produit industriel et le rendre attrayant, il faut cibler le volet esthétique, communication, hédonique, efficience, excellence, utilitaire et monétaire de la valeur perçue. Il serait intéressant dans ce travail d'accompagner les designers dans la conception de mèche de cheveu innovante et dans la communication publicitaire. D'autre part, la valeur perçue conduit à positionnement du produit et par ricochet à avoir fortement l'intention d'achat.

La limite majeure de cette recherche semble être d'ordre méthodologique et porte sur les entretiens. En dépit du fait que les répondants semblent « avoir joué le jeu » en parlant librement de leur consommation, nous savons bien que notre présence masculine a pu par moment intimider ou influencer, d'une manière ou d'une autre des interviewés et par conséquent biaiser peut être les résultats. Certaines répondantes ont parfois tendance à se minimiser ou à avoir une haute estime d'elles-mêmes, ce qui se dépeint sur leurs réponses.

Les voies de recherche future concernant cette recherche exploratoire nous emmène à proposer deux modèles à tester quantitativement. Le premier modèle est la valeur globale perçue dans l'utilisation des faux cheveux serait motivée par les valeurs de beauté, d'estime de soi, de plaisir, de gain de temps, de protection et de prix et le second serait l'analyse des déterminants de l'intention d'achat des faux cheveux.

\section{BIBLIOGRAPHIE}

[1] P. Aurier, Y. Evrard et G. N'Goala, « Comprendre et mesurer la valeur du point de vue du consommateur », Recherche et Applications en Marketing, 19, (3), pp.1-20, 2004.

[2] P. Aurier, Y. Evrard et G., «La valeur du produit du point de vue du consommateur », Actes des 12èmes Journées Nationales des I.A.E, pp.199-212, 1998.

[3] P. Aurier, Y. Evrard et G. N'Goala, «Validité discriminante et Prédictive des Composantes de la Relation », actes du 17 ème Congrès de l'Association Française de Marketing, Caen Basse -Normandie, 2001.

[4] P. Aurier et J. Passebois, « Comprendre les expériences de consommation pour mieux gérer la relation client », Décisions Marketing, 28, (4), pp. 43-52, 2002.

[5] P. Aurier, Y. Evrard et G. N'Goala, « Comprendre et mesurer la valeur du point de vue du consommateur », Recherche et Applications en Marketing, Vol. 19, No. 3, pp. 1-20, 2004. 
[6] E. N. Banister, et M. K. Hogg, «Negative symbolic consumption and consumers' drive for self-esteem: the case of the fashion industry», European Journal of Marketing, vol.38, n 7, pp. 850-868, 2004.

[7] N. Bernoussi et L. Sirieix, «L'impact des attributs et indicateurs de qualité sur le comportement d'achat d'un produit alimentaire : une approche exploratoire, par la valeur perçue et l'authenticité perçue: cas de l'huile d'olive d'Algérie », 2020. HAL Id: hal-02493533.

[8] J.-P. Brechet et A. Desreumaux, «Le thème de la valeur en sciences de gestion : représentations et paradoxes », XIVème Journées des IAE, NANTES, 28/29, avril 1998.

[9] E. Day, «The role of value in consumer satisfaction », Journal of Consumer Satisfaction, Dissatisfaction and Complaining Behavior, 15, (1), pp. 22-32, 2002.

[10] P. Dibie, « Ça décoiffe pas mal », Sociétés et Représentations, n³5, printemps, pp. 255-260, 2013.

[11] D. Djoma et V. Tsapi, «L'adoption des produits cosmétiques par les consommateurs camerounais : la forte incidence de la nouveauté perçue, de l'innovativité, de l'implication et des valeurs », African Sociological Review, vol. 16, n 1 , pp. 100-120, 2012

[12] Y. Evrard et P. Aurier, «Identification and validation of the components of the person-object relationship », Journal of Business Research, 37, (2), pp. 127-134, 1996.

[13] M.-L. Gavard-Perret, G. Gotteland, C. Haon et A. Jolibert « Méthodologie de la recherche en Sciences de Gestion », 2e édition, Pearson, 2012.

[14] E.C. Hirschman et M.B. Holbrook, « Hedonic Consumption : Emerging Concepts, Methods and Propositions », Journal of Marketing, 46, (3), pp. 92-101, 1982.

[15] M.B. Holbrook, «The nature of customer value: an axiology of service in the consumption experience, Service quality: new directions in theory and practice », Eds. R. Rust et R.L Oliver, Thousand Oaks, Sage Publications, 1994, pp. 21 71.

[16] M.B. Holbrook, « Consumer Value », Eds. Routledge, London, 1999.

[17] D.B. Holt, « How Consumers Consume : A Typology of Consumption Practices », Journal of Consumer Behavior, 22 , (1), pp. 1-16, 1995.

[18] M. A. Innocent, A. François-Lecompte et M. Le Gall-Ely, «La valeur de la maîtrise de la consommation électrique : multi-dimensionnalité et bivalence », Décisions Marketing, 83, pp. 11-28, 2016.

[19] R. A. Jones, «Méthodes de recherche en sciences humaines », De Boeck Université, Paris, 2000.

[20] E. Karababa et D. Kjeldgaard, « Value in marketing: Toward sociocultural perspectives », Marketing Theory, 14(1), pp. 119-127, 2014

[21] D. Katz, « The functional approach to the study of attitudes », Public Opinion Quarterly, 24, (2), pp. 163-204, 1960.

[22] A.W. Lai, « Consumer values, product benefits and customer value : a consumption behavior approach », Advances in Consumer Research, 22, éds. F.R. Kardes et M. Sujan, Provo, Utah, Association for Consumer Research, 1995, pp. 381-388.

[23] K. Lancaster, « A new approach to consumer theory », Journal of Political Economy, 74, pp. 132-157, 1966.

[24] E. Le Nagard et G. Michel, «Consommateurs, nous n'avons pas les mêmes », Décisions Marketing, 86, (2), pp. 5-7, 2017.

[25] A. Monjaret et F. Tamarozzi, « Pas de demi-mesure pour les miss : la beauté en ses critères », Ethnologie française 3, vol. 35, pp. 425-443, 2005. 
[26] A. Ouattara, «Marketing en Afrique, concepts et Applications », Edition Etudes Conseils et Star Editions, 2003 AbidjanBénin.

[27] J.-M. Peretti et A. Delannoy, « Le Marketing Ethnique, au croisement des sphères économiques, politiques et éthique », Management et Avenir, vol. 2, n³2, pp. 286-298, 2010.

[28] J. Perrin, « Analyse de la valeur et valeur économique des biens et services », Revue Française de Gestion Industrielle, Vol. 20, $\mathrm{N}^{\circ} 2,2001$.

[29] M.E. Porter, « L'avantage concurrentiel », Paris, Inter Editions, 1986.

[30] R. Mencarelli et A. Rivière «La valeur perçue en marketing ; perspectives théoriques et enjeux managériaux » Presses Universitaires de Provence (PUP) - Collection « Travail \& Gouvernance », 17 Juin 2021.

[31] A. Rivière, «Vers un modèle de formation de la valeur perçue d'une innovation: le rôle majeur des bénéfices perçus en amont du processus d'adoption », Recherche et Applications en Marketing, 30, (1), pp. 5-27, 2015.

[32] A. Rivière et R. Mencarelli, «Vers une clarification théorique de la notion de valeur perçue en marketing », Recherche et Applications en Marketing, 27, (3), pp. 97-123, 2012.

[33] K. Rondou, « Coiffures. Les cheveux dans la littérature et la culture françaises du xixe siècle », trad. de l'américain par Carol Rifelj et Camille Noiray, Paris, H. Champion, coll. Romantisme et modernités, 2014), p 312.

[34] J. N. Sheth, B. I. Newman, et B. Gross, « Consumption values and market choice: theory and applications », Cincinnati, Oh: Southwestern Publishing, 1991.

[35] J.B. Steenkamp, et H. Baumgartner, «The role of optimum stimulation level in exploratory consumer behavior », Journal of Consumer Research, 19, (3), pp. 434-448, 1992.

[36] R. B. Woodruff, « Customer value: the next source of competitive advantage », Journal of the academy of Marketing science, 25, (2), pp. 139-153, 1997.

[37] V.A. Zeithaml, «Consumer perceptions of price, quality, and value: a means-end model and synthesis of evidence », Journal of Marketing, 52, (3), pp. 2-22, 1988.

[38] https://www.youtube.com/watch?v=Qu3ESIV0-gg\&list=RDCMUCCihDvmcTRdmmV80wBznQOA\&index=2

[39] http://www.afriquefemme.com/fr/beaute/cheveux/2426-incroyables-les-femmes-africaines-depensent-environ-7milliards-par-an-pour-l-achat-des-faux-cheveux 